\title{
BMJ Open Rapid Deployment Aortic Replacement (RADAR) Registry in Spain: a protocol
}

\author{
Victor Bautista-Hernandez, ${ }^{1,2}$ Natalia Cal-Purriños, ${ }^{3}$ Jose M Arribas-Leal, ${ }^{4}$ \\ Manuel Carnero-Alcazar, ${ }^{5}$ Jose F Gutierrez-Diez, ${ }^{6}$ Jose J Cuenca-Castillo $^{1}$
}

To cite: Bautista-

Hernandez V, Cal-Purriños N, Arribas-Leal JM, et al. Rapid Deployment Aortic Replacement (RADAR) Registry in Spain: a protocol. BMJ Open 2017;7:e011437. doi:10.1136/bmjopen-2016011437

- Prepublication history for this paper is available online. To view these files please visit the journal online (http://dx.doi.org/10.1136/ bmjopen-2016-011437).

Received 9 February 2016 Revised 8 June 2016 Accepted 1 August 2016

\section{CrossMark}

For numbered affiliations see end of article.

Correspondence to Dr Victor Bautista-Hernandez; victor.bautista.hernandez@ sergas.es

\section{ABSTRACT}

Introduction: Rapid deployment valves (RDV) represent a newly introduced approach to aortic valve replacement which facilitates surgical implantation and minimally invasive techniques, shortens surgical times and shows excellent haemodynamic performance. However, evidence on their safety, efficacy and potential complications is mostly fitted with small-volume and retrospective studies. Moreover, no current guidelines exist. To improve our knowledge on this technology, The Rapid Deployment Aortic Replacement (RADAR) Registry will be established across Spain with the aim of assessing RDV outcomes in the real-world setting.

Methods and analysis: The RADAR Registry is designed as a product registry that would provide information on its use and outcomes in clinical practice. This multicentre, prospective, national effort will initially involve 4 centres in Spain. Any patient undergoing cardiac surgery for aortic valve replacement and receiving an RDV as an isolated operation or in combination with other cardiac procedures may be included. Participation is voluntary. Data collection is equal to information obtained during standard care and is prospectively entered by the participating physicians perioperatively and during subsequent follow-up visits. The primary outcome assessed is in-hospital and up to 5 years of follow-up, prosthetic valve functioning and clinical status. Secondary outcomes are to perform subgroup analysis, to compare outcomes with other existing approaches and to develop future clinical guidelines. The follow-up assessments are timed with routine clinical appointments. Dissociated data will be extracted and collectively analysed. Initial target sample size for the registry is 500 participants entered with complete follow-up information. Different substudies will be implemented within the registry to investigate specific populations undergoing aortic valve replacement.

Ethics and dissemination: The protocol is approved by all local institutional ethics committees. Findings will be shared by the participant hospitals, policymakers and the academic community to promote quality monitoring and efficient use of this technology.

\section{INTRODUCTION}

Aortic stenosis (AS) is the most frequent valvular disease in Western countries with an

\section{Strengths and limitations of this study}

The Rapid Deployment Aortic Valve Replacement (RADAR) Registry is a prospectively conducted multicentre national registry in which patients will be observed perioperatively and up to 1 year of follow-up in the real-world setting.

- No interference with the clinical decision-making process of physicians and patients.

- Clinical intervention and collected information equals the standard of care.

- Institutional biases may be difficult to control.

no concurrent control arm for comparison.

estimated prevalence between $3 \%$ and $23 \%$ of the elderly population. ${ }^{1}$ Moreover, with the current ageing of the western countries, its impact on the consumption of health resources is expected to increase. ${ }^{23}$ The prognosis of symptomatic severe AS is dismal with a 1 year mortality of $30-50 \% .^{4} 5$ Among elderly patients with severe AS, $75 \%$ of them have symptoms. Aortic valve replacement (AVR) has been and remains the gold standard for severe AS due to excellent early outcomes and long-term survival. ${ }^{6}$ However, 30 $40 \%$ of patients with severe AS are deemed inoperable due to high perioperative risk, significant comorbidities or advanced age.

Transcatheter aortic valve implantation (TAVI) was introduced in clinical practice in 2002. Since then, TAVI has proved to be superior to medical therapy in inoperable patients and also not inferior to surgical AVR in high-risk patients. ${ }^{8-10}$ Moreover, some groups have applied TAVI to intermediate-risk and even low-risk patients with controversial results. In general, comparison between TAVI and surgical AVR on those later patients, that is, patients in the 'grey zone', does not show significant differences in terms of mortality, although patients undergoing TAVI elicit a higher incidence of significant complications during follow-up such as aortic regurgitation and stroke. ${ }^{11-14}$ Results from the PARTNERS II trial conclude that in intermediate-risk patients, TAVI was similar to surgical AVR with 
respect to the primary end point of death or disabling stroke. ${ }^{15}$ Rosato et $a l^{16}$ have recently reported in patients with low operative risk a significantly better 3-year survival and freedom from major cardiac and cerebrovascular in patients undergoing surgical AVR compared with TAVI. In addition, controversy exists on the cost-effectiveness of TAVI. ${ }^{17}$ A recent study carried out in 19 European countries estimates an annual number of new TAVR candidates of 17712 , which would represent a cost of $\$ 13.7$ billion. ${ }^{18}$ Despite budgetary concerns, current clinical trials are evaluating TAVI for patients at intermediate and even low surgical risk. ${ }^{19-21}$

Rapidly evolving technological progress has resulted in innovative sutureless valves from conventional sutured valves. By avoiding or significantly reducing placement and tying of sutures after native valve removal and decalcification, these valves have been shown to facilitate minimally invasive approaches, shorten surgical times and improve costs. These effects may be of great interest, especially in high-risk patients, elderly 'fragile' patients and in those undergoing complex or concomitant procedures. ${ }^{22}$ Moreover, the additive beneficial effect of sutureless AVR through a minimally invasive approach may expand current indications to high-risk patients. However, owing to recent implementations in clinical practice, little evidence and even controversial data on the outcomes of sutureless valves exist. ${ }^{23}$

The rapid deployment Intuity valve (Edwards Lifesciences, Irvine, USA) received European Conformity (CE) approval in 2012. This valve is a stented trileaflet made out of bovine pericardium. Its design is based on the Perimount family of biological valves. A balloon expandable stainless steel cloth-covered frame is incorporated into the inflow aspect of the valve. After native aortic valve removal, the rapid deployment valves (RDV) is implanted with the aid of a delivery system, which allows for expansion of the frame with a balloon catheter within the left ventricular outflow tract. The expandable frame and the sewing ring assist to position, stabilise and seal the valve at implant. Three additional stitches (guiding sutures) are used at the nadir points of each sinus of Valsalva to facilitate valve position and further secure the valve.

While the initial results of this technology are partially reported, The Rapid Deployment Aortic Valve Replacement (RADAR) Registry will assess the evolving use and outcomes of RDV in the real-world setting. Moreover, the registry will allow comparison with existing and accepted procedures for patients with aortic valve disease in different risk settings and may help develop future clinical guidelines.

\section{OBJECTIVE}

The primary outcome assessed is to generate a national multicentre prospective database for patients undergoing AVR with an RDV and assess perioperative as well as up to 1 year of follow-up clinical outcomes and haemodynamic profiles by echocardiography.
Secondary objectives of this project will be to: (1) perform subgroup comparative analysis based on riskstratified patients, surgical approach (minimally invasive vs conventional approach), associated surgical procedures, age at surgery (eg, elderly), valve size and function; (2) identify potential surgical and prognostic risk factors; (3) compare the results of this population with reported outcomes of patients undergoing AVR with sutureless valves, conventional prosthesis or with transcatheter implanted valves; and (4) generate clinical guidelines for the use of this technology.

\section{METHODS}

\section{Study design}

This study is a multicentre, prospective, single-arm national registry study. The protocol has been approved by the local and regional ethics committee at the coordinating centre (Study \#: Xunta de Galicia/Conselleria de Sanidade: 2016/ 018). The four local participating centres have joined the protocol. This registry plans to recruit 500 consecutive patients who meet the inclusion criteria. Participation is fully voluntary and the intervention and data collection equals the standard of care for these patients. After the enrolment, all participants would be evaluated perioperatively and up to 1 year of follow-up approximately.

This study is conceptualised, designed and initiated by the Department of Cardiovascular Surgery at Xerencia de Xestión Integrada de A Coruña (XXIAC) and the Congenital and Structural Heart Disease research group at Instituto de Investigación Biomédica de A Coruña (INIBIC). This institution is the coordinating centre and provides with the promoter (JJC-C) and the coordinator and primary investigator (VB-H). A lawyer with experience on health research was designed to accomplish ethics and privacy standards during the development and data gathering of the registry (NC-P). Initially, three additional participant centres from across Spain with former experience with sutureless valves and more than $20 \mathrm{RDV}$ AVR operations performed were invited to participate. Hospital Clínico San Carlos (Madrid), Hospital Marqués de Valdecilla (Santander) and Hospital Virgen de la Arrixaca (Murcia) agreed to join the protocol. A primary local investigator was appointed at each participating centre (JMA-L, MC-A, JFG-D). Selection of institutions with former experience with this technology will minimise bias in patient demographics, surgical indications and approaches, technical skills and clinical care, thus allowing for widespread acceptance of the results. The RADAR Registry is an open project, and therefore institutions fulfilling the above specified requirements may join the protocol during the recruitment phase. Moreover, hospital with $<20$ implants could report outcomes but those patients will be analysed on a separate basis.

\section{Participants}

Any patients undergoing AVR with an RDV will be included in the study regardless of the underlying 
disease, surgical approach, age at surgery or concomitant surgery. Participation is voluntary. Recruitment is non-competitive and thus participating centres do not have to alter their practice.

\section{Informed consent}

Written informed consent will be obtained from the patient or patient's authorised representative prior to study inclusion. The study will be conducted in accordance with the provisions of the Declaration of Helsinki and the Biomedicine Oviedo Convention.

\section{Data collection and management}

After the approval of the RADAR Registry, patients will be recruited in a prospective manner. It is expected to enrol $\sim 100$ patients per year among the participating centres and thus, the study period will be from 2016 to 2021 where 500 consecutive patients have been enrolled and follow-up completed. The recruitment phase is expected to be closed in 2020. The duration of data collection may be renewed and extended at the primary investigator's request and agreement.

A dissociated standard data form has been created to obtain relevant demographic, surgical, clinical and echocardiographic information from medical records. All institutions involved will prospectively collect the requested data in the standardised form. Then data will be dissociated and sheets submitted by the primary investigators to the coordinating centre for further analysis. Data will be stored in a secure database. At registry completion, data will be analysed. Interim annual evaluations are also planned.

Patients having RDV-AVR formerly to the approval date of the RADAR Registry will undergo retrospective collection of the data by all participant centres. Results obtained from the national retrospective database will be used as a platform to evaluate the initial results of this technology and the surgical practice evolution overtime. Data from this database will be reported independently from the results obtained out of the prospective registry.

\section{Data elements}

Participants will undergo assessments during surgical admission, both in the early postoperative period and at 1 year of follow-up approximately. Information to be collected is meant to specifically address the objectives of the registry.

Summarised variables compiled within the RADAR Registry will include:

1. General information and demographics: age, weight, height, gender, smoking, high blood pressure, dyslipidaemia, peripheral arterial disease, chronic obstructive pulmonary disease, stroke, ischaemic heart disease, pulmonary hypertension, chronic respiratory disease and Valve Academic Research Consortium (VARC) criteria.

2. Risk assessment information: New York Heart Association (NYHA) class, emergent surgery,
EuroSCORE I and EuroSCORE II, Society of Thoracic Surgeons (STS) score.

3. Surgical information: surgical approach, conversion from minimally invasive approach, cross-clamp time, cardiopulmonary by-pass time, prosthesis size, implantation failure, other intraoperative complications, concomitant surgery.

4. Clinical information:

- Preoperative: heart rhythm, haemoglobin and creatinine, Risk, Injury, Failure, Loss of kidney function, and End-stage kidney disease (RIFLE)/acute kidney injury (AKIN) score.

- Perioperative: redo for bleeding or any cause, cardiac tamponade, blood transfusion, postoperative atrial fibrillation, permanent pacemaker implantation, wound infection/ mediastinitis, myocardial infarction, heart rhythm, intensive care unit and in-hospital stay and discharge status.

- Postdischarge/follow-up: readmission with cause, NYHA class, complications and clinical status at most recent-follow-up.

5. Echocardiographic information: left ventricular ejection fraction, right ventricular function, aortic annulus diameter, sinotubular junction diameter, ascending aorta diameter, peak transvalvular gradient, mean transvalvular gradient, other associated structural heart disease and prosthetic perivalvular or intravalvular insufficiency with grade and location, effective valve area.

\section{Statistical analysis}

Statistical analysis will be conducted on an as-treated basis. Continuous variables will be expressed as means and SDs, and discrete variables as frequencies and percentages. Statistical comparison will be performed using the $\chi^{2}$ or Fisher's exact tests for categorical variables. The Student's t and the Mann-Whitney tests will be used for continuous variables. To compensate for intrinsic heterogeneity between patient subgroups, propensityscored matching analysis will be used. A $\mathrm{p}$ value $<0.05$ will be considered significant.

\section{DISCUSSION}

Recently introduced RDV technology represents an innovative and promising therapy for patients with severe AS which may improve outcomes, expand surgical indications and reduce costs in this high prevalence population. However, given its recent introduction in the field of structural heart disease, little is known about the use, outcomes, haemodynamic profile and complications of this prosthesis. Actually, current evidence shows significant limitations, mainly because it is mostly based on small-volume and retrospective studies, with two prospective trials performed until now.

In 2013, initial results of the prospective, single-arm TRITON trial were reported. ${ }^{24}$ RDV-AVR was implanted 
successfully in 146 patients with a mean age at surgery of 75.5 years. Concomitant surgery was performed in $41.1 \%$ of cases and minimally invasive approaches were undertaken in $48.8 \%$ of cases. Early mortality was $1.4 \%$ and cumulative survival was $92.5 \%$ at a mean follow-up of 9.8 months. Cross-clamp time for isolated AVR was $41.1 \mathrm{~min}$. The valves showed good haemodynamic performance by echocardiography at 3 months and 1 year of follow-up. This was the first report of safeness and efficacy of the valve. In 2014, 3-year follow-up results of the TRITON trial were published showing significant left ventricular mass regression and maintained excellent haemodynamics over time. Patient-prosthesis mismatch was found in $3 \%$ of patients. ${ }^{25}$ This was the first trial reporting RDV as a feasible, safe and efficacious method for AVR.

Results of the CADENCE-MIS trial were reported in $2015 .{ }^{26}$ This study is a prospective, multicentre randomised trial comparing patients having an isolated RDV-AVR through upper hemisternotomy $(n=51)$ with patients undergoing AVR with a conventional stented bioprosthesis through full sternotomy $(n=49)$. Patients having an RDV showed a significant reduction in crossclamp times, lower mean transvalvular gradients and lower prevalence of patient-prosthesis mismatch when compared with patients with a conventional bioprosthesis. Authors concluded that RDV may facilitate the performance of minimally invasive surgery.

Some other studies have initially reported on RDV regarding: potential benefits of this technology in patients with a small aortic annulus; ${ }^{27}$ technical issues when severe calcification of the sinotubular junction is present $^{28}$ and application to patients having surgery under emergency cardiogenic shock. ${ }^{29}$ However, those and other pending issues may be answered by prospective registries. Actually, in an effort to further elucidate the short-term and long-term outcomes of sutureless valves (including RDV), the International Valvular Surgery Study Group has set up a Sutureless Aortic Valve Replacement International Registry which will be completed in 2021. The majority of participating centres are located in Germany and Italy.

In conclusion, owing to the current paucity of studies and the ongoing competition of RDV with existing wellaccepted treatments for aortic valve disease, the large cohort of patients enrolled in the RADAR Registry will provide robust clinical evidence on the outcomes of $\mathrm{RDV}$, as well as allow for comparisons among the participants and with other registries (TAVI and sutureless) and hopefully help to develop clinical guidelines on its use.

\footnotetext{
Author affiliations

${ }^{1}$ Department of Cardiovascular Surgery, Xerencia de Xestión Integrada A Coruña (XXIAC), A Coruña, Spain

${ }^{2}$ Congenital and Structural Heart Disease, Instituto de Investigación Biomédica A Coruña (INIBIC), A Coruña, Spain

${ }^{3}$ Fundación Profesor Novoa Santos, Xerencia de Xestión Integrada A Coruña (XXIAC), A Coruña, Spain
}

${ }^{4}$ Department of Cardiovascular Surgery, Hospital Virgen de la Arrixaca, Murcia, Spain

${ }^{5}$ Department of Cardiovascular Surgery, Hospital Clínico de San Carlos, Madrid, Spain

${ }^{6}$ Department of Cardiovascular Surgery, Hospital Marqués de Valdecilla, Santander, Spain

Contributors All authors have contributed to the design and development of the protocol. Concept and supervision of the protocol was conducted by JJC-C. Design of the protocol and drafting of the manuscript was done by VB-H. Ethics, legal and technical support was provided by NC-P. Critical revision of the protocol was performed by JMA-L, MC-A and JFG-D.

Competing interests None declared.

Patient consent Obtained.

Ethics approval Official IRB of the Region of Galicia (Spain).

Provenance and peer review Not commissioned; externally peer reviewed.

Data sharing statement Since this is a prospective protocol, the data are not gathered or published. Some of the authors have presented in local meetings and journals the retrospective experience with this prostheses but on a single institution basis.

Open Access This is an Open Access article distributed in accordance with the Creative Commons Attribution Non Commercial (CC BY-NC 4.0) license, which permits others to distribute, remix, adapt, build upon this work noncommercially, and license their derivative works on different terms, provided the original work is properly cited and the use is non-commercial. See: http:// creativecommons.org/licenses/by-nc/4.0/

\section{REFERENCES}

1. Ross J Jr, Braunwald E. Aortic stenosis. Circulation 1968;38:61-7.

2. Nkomo VT, Gardin JM, Skelton TN, et al. Burden of valvular heart diseases: a population-based study. Lancet 2006;368:1005-11.

3. lung $B$, Vahanian A. Epidemiology of valvular heart disease in the adult. Nat Rev Cardiol 2011;8:162-72.

4. Varadarajan P, Kapoor N, Bansal RC, et al. Clinical profile and natural history of 453 nonsurgically managed patients with severe aortic stenosis. Ann Thorac Surg 2006;82:2111-15.

5. Vahanian A, Otto CM. Risk stratification of patients with aortic stenosis. Eur Heart J 2010;31:416-23.

6. Brown JM, O'Brien SM, Wu C, et al. Isolated aortic valve replacement in North America comprising 108,687 patients in 10 years: changes in risks, valve types, and outcomes in the Society of Thoracic Surgeons National Database. J Thorac Cardiovasc Surg 2009;137:82-90.

7. Brennan JM, Edwards $\mathrm{FH}$, Zhao $\mathrm{Y}$, et al. Long-term survival after aortic valve replacement among high-risk elderly patients in the United States: insights from the Society of Thoracic Surgeons Adult Cardiac Surgery Database, 1991 to 2007. Circulation 2012;126:1621-9.

8. Makkar RR, Fontana GP, Jilaihawi $\mathrm{H}$, et al. Transcatheter aortic-valve replacement for inoperable Severe Aortic Stenosis. N Engl J Med 2012;366:1696-704.

9. Leon MB, Smith CR, Mack M, et al. Transcatheter aortic-valve implantation for aortic stenosis in patients who cannot undergo surgery. N Engl J Med 2010;363:1597-697.

10. Kapadia SR, Tuzcu EM, Makkar RR, et al. Long-term outcomes of inoperable patients with aortic stenosis randomly assigned to transcatheter aortic valve replacement or standard therapy. Circulation 2014:130:1483-92.

11. Latib A, Maisano F, Bertoldi L, et al. Transcatheter vs surgical aortic valve replacement in intermediate-surgical-risk patients with aortic stenosis: a propensity score-matched case-control study. Am Heart $J$ 2012;164:910-7.

12. Piazza N, Kalesan B, van Mieghem M, et al. A 3-center comparison of 1-year mortality outcomes between transcatheter aortic valve implantation and surgical aortic valve replacement on the basis of propensity score matching among intermediate-risk surgical patients. $J$ Am Coll Cardiol Intv 2013;6:443-51.

13. D'Errigo P, Barbanti M, Ranucci M, et al. Transcatheter aortic valve implantation versus surgical aortic valve replacement for severe aortic stenosis: results from an intermediate risk propensity-matched population of the Italian OBSERVANT study. Int J Cardiol 2013;167:1945-52. 
14. Wenaweser P, Stortecky S, Schwander S, et al. Clinical outcomes of patients with estimated low or intermediate surgical risk undergoing transcatheter aortic valve implantation. Eur Heart $J$ 2013;34:1894-905.

15. Leon MB, Smith CR, Mack MJ, et al. Transcatheter or surgical aortic-valve replacement in intermediate-risk patients. N Engl J Med 2016;374:1609-20.

16. Rosato S, Santini F, Barbanti M, et al. Transcatheter aortic valve implantation compared with surgical aortic valve replacement in low-risk patients. Circ Cardiovasc Interv 2016;9:e003326. (in press).

17. Neyt $M$, Van Brabandt $H$, Devriese $S$, et al. A cost-utility analysis of transcatheter aortic valve implantation in Belgium: focusing on a well-defined and identifiable population. BMJ Open 2012;2: e001032 47.

18. Osnabrugge RL, Mylotte D, Head SJ, et al. Aortic stenosis in the elderly. Disease prevalence and number of candidates for transcatheter aortic valve replacement: a meta-analysis and modeling study. J Am Coll Cardiol 2013;62:1002-12.

19. Kappetein AP, Head SJ, Généreux P, et al. Updated standardized endpoint definitions for transcatheter aortic valve implantation: the Valve Academic Research Consortium-2 consensus document. J Am Coll Cardiol 2012;60:1438-54.

20. Osnabrugge RL, Head SJ, Bogers AJ, et al. Patient selection for transcatheter aortic valve replacement: what does the future hold? Expert Rev Cardiovasc Ther 2012;10:679-81.

21. Thyregod HG, Steinbrüchel DA, Ihlemann N, et al. Transcatheter versus surgical aortic valve replacement in patients with severe aortic stenosis. 1-year results from the all-comers NOTION randomized clinical trial. J Am Coll Cardiol 2015;65:2184-94.
22. Ranucci M, Frigiola A, Menicanti L, et al. Aortic cross-clamp time, new prostheses, and outcome in aortic valve replacement. J Heart Valve Dis 2012;21:732-9.

23. Forcillo J, Bouchard D, Nguyen A, et al. Perioperative outcomes with sutureless versus stented biological aortic valves in elderly persons. $J$ Thorac Cardiovasc Surg 2016;151:1629-36.

24. Kocher AA, Laufer G, Haverich A, et al. One-year outcomes of the surgical treatment of aortic stenosis with a next generation surgical aortic valve (TRITON) trial: a prospective multicenter study of rapid-deployment aortic valve replacement with the EDWARDS INTUITY valve system. J Thorac Cardiovasc Surg 2013;145:110-15.

25. Haverich A, Wahlers TC, Borger MA, et al. Three-year hemodynamic performance, left ventricular mass regression, and prosthetic-patient mismatch after rapid deployment aortic valve replacement in 287 patients. J Thorac Cardiovasc Surg 2014;148:2854-60.

26. Borger MA, Moustafine V, Conradi L, et al. A randomized multicenter trial of minimally invasive rapid deployment versus conventional full sternotomy aortic valve replacement. Ann Thorac Surg 2015;99:17-25.

27. Theron A, Gariboldi V, Grisoli D, et al. Rapid deployment of aortic bioprosthesis in elderly patients with small aortic annulus. Ann Thorac Surg 2016;101:1434-41.

28. Wahlers TC, Rahmanian PB. Rapid deployment valve implantation using the EDWARDS Intuity Valve System: a word of caution regarding sizing in calcified sinotubular junctions. Thorac Cardiovasc Surg 2015;63:504-7.

29. Martinelli GL, Braccio M, Cotroneo A, et al. Treatment of cardiogenic shock in severe aortic stenosis with the Edwards INTUITY valve. Ann Thorac Surg 2014;98:e107-8. 